\title{
HÖLDER CONDITIONS FOR GAUSSIAN PROCESSES WITH STATIONARY INCREMENTS
}

\author{
BY \\ M. B. MARCUS
}

Introduction. Hölder conditions, both uniform and local, will be obtained for a wide class of separable, real-valued Gaussian processes with stationary increments. Let $X(t)$ be such a process with $E\{X(t)\}=0$ and $E\left\{(X(t))^{2}\right\}<\infty$. These processes will be characterized by their incremental covariance, i.e., the function $\sigma^{2}(h)=E\left\{(X(t+h)-X(t))^{2}\right\}$. It is assumed that $\sigma^{2}(h) \rightarrow 0$ as $h \rightarrow 0$; we shall exclude the trivial case when $\sigma^{2}(h)$ is identically zero. Since the continuity properties of the paths of these processes depend only on $X(t+h)-X(t)$ for small $h$, we are only concerned with the function $\sigma^{2}(h)$ for $h \in[0, \delta]$ for some $\delta>0$. The major portion of our results apply to processes for which $\sigma^{2}(h)$ is concave for $h \in[0, \delta]$.

This study is motivated by the well-known results for Brownian motion, the law of the iterated logarithm and Paul Levy's uniform Hölder condition. Therefore, representing a real-valued, separable Gaussian process with stationary increments by the corresponding function $\sigma^{2}(h)$, we seek functions $f(h)$ and $f(h)$ for which the following events have probability 1 :

$$
\begin{gathered}
C_{0} \leqq \limsup _{h \rightarrow 0} \frac{|X(t+h)-X(t)|}{\left(2 \sigma^{2}(h) f(h)\right)^{1 / 2}} \leqq C_{1}, \\
C_{0}^{\prime} \leqq \lim _{\left|t-t^{\prime}\right|=h \rightarrow 0 ; 0 \leqq t, t^{\prime} \leqq 1} \frac{\left|X\left(t^{\prime}\right)-X(t)\right|}{\left(2 \sigma^{2}(h) f^{f}(h)\right)^{1 / 2}} \leqq C_{1}^{\prime},
\end{gathered}
$$

where $C_{0}, C_{0}^{\prime}>0 ; C_{1}, C_{1}^{\prime}<\infty$.

In $\S 1$, we consider those functions $f(h)$ and $f(h)$, which naturally depend upon $\sigma^{2}(h)$, for which the ratios in $(0.1)$ and $(0.2)$ can be bounded below by some $C_{0}$ and $C_{0}^{\prime}>0$. This is done for all processes for which $\sigma^{2}(h)$ is concave in [C, $\left.\delta\right]$ for some $\delta>0$. It turns out that in these cases, the Chung-Erdös lemma [1], [2] enables us to extend techniques usually used with independent random variables.

In $\$ 2$, we consider the upper bound. Basing our work on a lemma by Fernique [3], we find functions $f(h)$ and $f^{7}(h)$ (not necessarily the same as those used in determining the lower bound) for which the ratios in (0.1) and (0.2) can be bounded above by constants $C_{1}, C_{1}^{\prime}<\infty$. This is done for all processes that are known to be continuous (i.e., that satisfy Fernique's requirements).

In $\S 3$, we are concerned with those processes for which the functions $f(h)$ and $f(h)$ that enable us to obtain nonzero lower bounds for $(0.1)$ and $(0.2)$ also enable us

Received by the editors May 11, 1967. 
to obtain noninfinite upper bounds. In other words, we are concerned with those processes for which we can give the appropriate $f(h)$ and $\tilde{f}(h)$ so that $(0.1)$ and (0.2) are satisfied with probability 1.

In Theorem 5 regularly varying functions are used. A function is said to be regularly varying if it can be put in the form $x^{p} L(x), p$ a real number, where $L(x)>0$ is slowly varying, i.e., $\lim _{x \rightarrow \infty} L(t x) / L(x)=1$ for any $t>0$. We obtain the following theorems where $g(\log 1 / h)$ is defined as $-\log \sigma(h)$ and consequently $1 / g^{\prime}(\log 1 / h)=\sigma(h) / h \sigma^{\prime}(h)$ :

THEOREM 5. Let $X(t)$ be a separable, real-valued Gaussian process with stationary increments for which $\sigma^{2}(h)$ is concave for $h \in[0, \delta]$ for some $\delta>0$. Assume that $1 / g^{\prime}(s)$ is a regularly varying function of $s$ such that $\log s \leqq 1 / g^{\prime}(s) \leqq \beta s, \beta<1$. Then the probability of event $(0.1)$ is 1 if the function $f(h)$ is taken either as $1 / g^{\prime}(\log 1 / h)$ or $[\sigma(h)]^{-1} \int_{0}^{h}(\sigma(u) / u) d u$.

If $1 / g^{\prime}(s)$ is also monotonic as $s \rightarrow \infty$, then it need only be bounded above, i.e., $1 / g^{\prime}(s) \leqq \beta s, \beta<1$. Equation (0.1) holds with $f(h)$ replaced by

$$
f^{*}(h)=\max \left(\log _{2} 1 / h, f(h)\right) .
$$

THEOREM 6. With $X(t)$ and $\sigma^{2}(h)$ as above, assume that $1 / g^{\prime}(\log 1 / h)=o\left(\log _{2} 1 / h\right)$, $\left(\log _{2} 1 / h \equiv \log (\log 1 / h)\right)$ and $\sigma^{2}(h) \leqq h^{2 \alpha}$ for some $\alpha>0$. The probability of event $(0.1)$ is 1 when $f(h)=\log _{2} 1 / h$ and $C_{0}=C_{1}=1$.

THEOREM 7. With $X(t)$ and $\sigma^{2}(h)$ as above assume that $1 / g^{\prime}(\log 1 / h)=o(\log 1 / h)$. The probability of the event $(0.2)$ is 1 when $f(h)=\log 1 / h$ and $C_{0}^{\prime}=C_{1}^{\prime}=1$.

COROLlary 3. If the restriction on $1 / g^{\prime}(\log 1 / h)$ is relaxed to $1 / g^{\prime}(\log 1 / h)$ $<a \log 1 / h(a<2)$, the probability of $(0.2)$ is still 1 when $\tilde{f}(h)=\log 1 / h$ but now $C_{0}^{\prime}=1$ while $C_{1}^{\prime}=$ Const $(2-a)^{-1}\left(\right.$ i.e., the upper bound contains the factor $\left.(2-a)^{-1}\right)$.

These theorems and the corollary provide the following examples of Hölder conditions for Gaussian processes with stationary increments.

$$
\begin{array}{llll}
\sigma^{2}(h)=h^{2 \alpha} & (\alpha<1) & f(h)=\log _{2} 1 / h & \tilde{f}(h)=\log 1 / h \\
\sigma^{2}(h)=e^{-(\log 1 / h)^{\gamma}} & (0<\gamma<1) & f(h)=(\log 1 / h)^{1-\gamma} & f(h)=\log 1 / h \\
\sigma^{2}(h)=1 /(\log 1 / h)^{a} & (a>1) & f(h)=\log 1 / h & \tilde{f}(h)=\log 1 / h .
\end{array}
$$

(Keep in mind that we are concerned only with $\sigma^{2}(h)$ for $h \in[0, \delta]$ for some $\delta>0$. The existence of these processes will be discussed in $\S 1$.)

Many other results related to the theorems mentioned are included in the body of this paper.

I am deeply indebted to Daniel Ray, under whose direction this work was initiated, for recognizing that the lemmas of Chung-Erdös and Fernique could be used to study the local behavior of Gaussian processes and for providing considerable assistance as the study progressed. I would also like to thank the referee for his many helpful comments. 
1. Lower bounds. Lower bounds are obtained for the almost sure Hölder conditions at a point and for the almost sure uniform Hölder conditions for a wide class of separable, real-valued Gaussian processes with stationary increments.

The point of departure of this work is a version of the Borel-Cantelli lemma due to Chung and Erdös [1] and a lemma by Chung, Erdös and Sirao [2] on Gaussian random variables. Since we require only simplified versions of these lemmas for this paper, simpler proofs can be given. For this reason and for the sake of the completeness of this paper, we shall prove restricted versions of the lemmas cited as Lemmas 1 and 2.

LEMMA 1. Let $\left\{B_{j}\right\}, j=1,2, \ldots$, be an infinite collection of events on a probability measure space such that $P\left(B_{j}, B_{k}\right) \leqq P\left(B_{j}\right) P\left(B_{k}\right), j \neq k$. If $\sum_{j=1}^{\infty} P\left(B_{j}\right)=\infty$ it follows that $P\left(B_{j}\right.$ infinitely often $)=1$. $\left(\right.$ Abbreviated $P\left(B_{j}\right.$ i.o. $\left.)=1\right)$.

Proof. The proof begins with an application of the Schwarz inequality. Let $\chi_{j}$ be the characteristic function of $B_{j}$, then

$$
\left[\sum_{j=n}^{m} P\left(B_{j}\right)\right]^{2}=\left[E\left(\sum_{j=n}^{m} \chi_{j}\right)\right]^{2} \leqq E\left\{\left(\sum_{j=n}^{m} \chi_{j}\right)^{2}\right\} P\left(\bigcup_{j=n}^{m} B_{j}\right) .
$$

It follows that

$$
\left[\sum_{j=n}^{m} P\left(B_{j}\right)\right]^{2} \leqq\left[\sum_{j=n}^{m} P\left(B_{j}\right)+\sum_{j \neq k=n}^{m} P\left(B_{j}, B_{k}\right)\right] P\left(\bigcup_{j=n}^{m} B_{j}\right)
$$

and

$$
P\left(\bigcup_{j=n}^{m} B_{j}\right) \geqq \frac{1}{\frac{1}{\sum_{j=n}^{m} P\left(B_{j}\right)}+1} .
$$

For any $\varepsilon>0$ we can choose a sequence of integers $\left\{n_{k}\right\}$ such that $P\left(\bigcup_{j=n_{k}}^{n_{k}+1} B_{j}\right)$ $\geqq 1-\varepsilon$. It follows that for any $n$ we can find an $n_{k} \geqq n$ so that $P\left(\bigcup_{j \geq_{n}} B_{j}\right) \geqq P\left(\bigcup_{j=n_{k}}^{n_{k}} B_{j}\right) \geqq 1-\varepsilon$. Since this is true for any $\varepsilon>0$ we get $\lim _{n \rightarrow \infty} P\left(\bigcup_{j \geqq n} B_{j}\right)=1$. Applying the monotone convergence theorem, this lemma is proved.

Lemma 1 will also be used in the form given in the following corollary. The proof of this corollary is almost identical to the proof of the lemma.

Corollary 1. Let $\mathscr{B}_{n}=\bigcup_{j=1}^{n} B_{j, n}$ and suppose that $P\left(B_{j, n}, B_{k, n}\right) \leqq P\left(B_{j, n}\right)$ - $P\left(B_{k, n}\right)$ for $j \neq k$. Then if $\lim _{n \rightarrow \infty} \sum_{j=1}^{n} P\left(B_{j, n}\right)=\infty$ it follows that $\lim \inf P\left(\mathscr{B}_{n}\right)=1$.

In Lemma 2 we show that certain events associated with negatively correlated Gaussian random variables have the property that the probability of a joint event is bounded above by the product of the probabilities of each event.

LEMMA 2. Let $X$ and $Y$ be two Gaussian random variables with mean zero such that $E\{X Y\} \leqq 0$. Then $P(X \geqq a, Y \geqq b) \leqq P(X \geqq a) P(Y \geqq b)$ where $a, b \geqq 0$. 
Proof. The author is grateful to the referee for this simple proof: We can write $Y=Z-c X$ where $Z$ is independent of $X$ and $c=-E\{X Y\} \mid E\left\{X^{2}\right\} \geqq 0$. We have

$$
\begin{aligned}
P(X \geqq a, Y \geqq b) & \leqq P(X \geqq a, Z \geqq b+c a) \leqq P(X \geqq a) P(Z \geqq b) \\
& \leqq P(X \geqq a) P(Y \geqq b)
\end{aligned}
$$

where the last inequality makes use of the fact that $E\left(Z^{2}\right) \leqq E\left(Y^{2}\right)$.

We will now display negatively correlated Gaussian random variables obtained from certain Gaussian processes with stationary increments. These are the processes with which we will be concerned in Theorems 1 and 2 .

Let $X(t)$ be a real-valued Gaussian process with stationary increments for which $E\{X(t)\}=0$ and $E\left\{(X(t))^{2}\right\}<\infty$. We require that

$$
E\left\{(X(t+h)-X(t))^{2}\right\}=\sigma^{2}(h) \rightarrow 0 \text { as } h \rightarrow 0 .
$$

Just as in the case when $X(t)$ is stationary, this condition implies that $\sigma^{2}(h)$ is continuous for all $h$. (We utilize the additional fact, which is also implied by (1.1), that $E\left\{(X(t))^{2}\right\}$ is continuous.) The covariance of $X(t)$ is given by

$$
\Gamma(t, s)=E\{X(t) X(s)\}=\frac{1}{2}\left[E\left\{X(t)^{2}\right\}+E\left\{X(s)^{2}\right\}-\sigma^{2}(|t-s|)\right] .
$$

Note that (1.1) implies that $\Gamma(t, s)$ is continuous in each variable.

Consider a real-valued Gaussian process $X(t)$ for which the function $\sigma^{2}(h)$ is concave for $h \in[0, \delta]$ for some $\delta>0$. Nonoverlapping increments of this process are negatively correlated. To see this, simply compute the following using (1.1a) (the sequence $\left\{t_{k}\right\} \rightarrow 0$, also without loss of generality, assume $j<k$ ):

$$
\begin{aligned}
2 E\left\{\left(X\left(t_{j+1}\right)-X\left(t_{j}\right)\right)\left(X\left(t_{k+1}\right)-X\left(t_{k}\right)\right)\right\} & \\
= & -\left[\sigma^{2}\left(t_{j}-t_{k}\right)-\sigma^{2}\left(t_{j+1}-t_{k}\right)\right]+\left[\sigma^{2}\left(t_{j}-t_{k+1}\right)-\sigma^{2}\left(t_{j+1}-t_{k+1}\right)\right] \leqq 0 .
\end{aligned}
$$

Note that the differences in the arguments of the pairs of terms in the brackets are both $t_{j+1}-t_{j}$. Since $t_{j}-t_{k}<t_{j}-t_{k+1}$ and because of the concavity of $\sigma^{2}(h)$ the inequality is obtained.

The question arises of whether there are Gaussian processes with stationary increments that satisfy $(1.1)$ for $\sigma^{2}(h)$ concave for $h \in[0, \delta]$. It is well known (see [4, Chapter IV, Exercise 13]) that a real, even, continuous function $g(u)$, with $g(0)=1$ and $g(u) \rightarrow 0$ as $u \rightarrow \infty$, which is convex on $[0, \infty]$, is a characteristic function and hence defines a stationary Gaussian process. Let us consider any continuous, concave function $\sigma^{2}(h)$ defined for $h \in[0, \delta]$. As long as $\sigma^{2}(h)$ is not identically zero we can choose a $\delta_{1}$ small enough so that $\sigma^{2}\left(\delta_{1}\right)>0$ and $\sigma^{\prime}\left(\delta_{1}\right)>0$. Consider the function $\frac{1}{2}\left(1-\sigma^{2}(h)\right)$ for $h \in\left[0, \delta_{1}\right]$; this is a continuous convex function on $\left[0, \delta_{1}\right]$, and it can be extended to an even continuous function $\Gamma(h)$ defined on $(-\infty, \infty)$ and convex on $[0, \infty]$ such that $\Gamma(h) \rightarrow 0$ as $h \rightarrow \infty$. The function $\Gamma(h)$ is the covariance of a stationary Gaussian process. This process satisfies (1.1) for $\sigma^{2}(h), h \in\left[0, \delta_{1}\right]$. Furthermore, this process can be used to obtain nonstationary processes with stationary increments for which (1.1) is also satisfied. 
In other words, to any continuous concave function $\sigma^{2}(h)$ on $[0, \delta]$ such that $\sigma(0)=0$, there corresponds a Gaussian process $X(t)$ with stationary increments such that $E\left\{(X(t+h)-X(t))^{2}\right\}=\sigma^{2}(h), h \in\left[0, \delta_{1}\right], 0<\delta_{1} \leqq \delta$.

Let $\sigma(h)=e^{-g(\log 1 / h)}$. The derivative of the function $g$ plays an important role in the results of this paper. Note that

$$
1 / g^{\prime}(\log 1 / h)=\sigma(h) / h \sigma^{\prime}(h) \geqq 1
$$

because $\sigma^{2}(h)$ concave implies that $\sigma(h)$ is concave.

The following properties also follow from the concavity of $\sigma(h)$ :

REMARK 1. At those points where $d / d s\left[1 / g^{\prime}(s)\right]$ exists, $d / d s\left[1 / g^{\prime}(s)\right]<1 / g^{\prime}(s)$.

REMARK 2. At the points of discontinuity of $1 / g^{\prime}(s),\left(1 / g^{\prime}\left(s^{+}\right)-1 / g^{\prime}(s)\right)<0$. This follows because $\sigma\left(e^{-s}\right) / e^{-s}$ is a continuous increasing function in $s$ whereas $1 / \sigma^{\prime}\left(e^{-s}\right)$ is decreasing. Since $1 / \sigma^{\prime}\left(e^{-s}\right)$ is not necessarily continuous $\sigma\left(e^{-s}\right) / e^{-s} \sigma^{\prime}\left(e^{-s}\right)$ can have jumps, but they are all negative.

REMARK 3. A necessary and sufficient condition that $\sigma^{2}(h) \log 1 / h$ decreases as $h \rightarrow 0$ is that $1 / g^{\prime}(\log 1 / h) \leqq 2 \log 1 / h$.

In Theorem 1 we obtain lower bounds for the Hölder condition for Gaussian processes with stationary increments. Several lower bounds are given; the first two are valid for all the processes under consideration. The third is sharper but it holds only when an additional condition is imposed on the processes.

THEOREM 1. Let $X(t)$ be a separable, real-valued Gaussian process with stationary increments for which $E\left\{(X(t+h)-X(t))^{2}\right\}=\sigma^{2}(h)$ where $\sigma^{2}(h)$ is concave for $h \in[0, \delta]$ for some $\delta>0$. Define $g(\log 1 / h)=-\log \sigma(h)$. Then

$$
P\left\{\limsup _{h \rightarrow 0} \frac{|X(t+h)-X(t)|}{\left(2 \sigma^{2}(h) f_{i}(h)\right)^{1 / 2}}>C_{i}\right\}=1,
$$

$i=1, \ldots, 3$, where the $f_{i}$ can be any one of the following functions. (The processes are subjected to whatever additional conditions are listed. The constants $C_{i}$ will be given in the proof.)

Case 1. $f_{1}(h)=\log \log 1 / h$.

Case 2. $f_{2}(h)=1 / 2 g^{\prime}(\log 1 / h)$.

Case 3.

$$
f_{3}(h)=\max \left\{\log \log 1 / h, \frac{1}{2 g^{\prime}(\log 1 / h)}\right\} \quad \text { if } \frac{1}{g^{\prime}(\log x)}
$$

increases monotonocially as $x \rightarrow \infty$.

Proof. Consider the event

$$
B_{k}=\left\{\frac{X\left(t+h_{k}\right)-X\left(t+h_{k+1}\right)}{\left(2 \sigma^{2}\left(h_{k}\right) f_{i}\left(h_{k}\right)\right)^{1 / 2}}>C_{1, i}\right\}
$$

where $h_{\kappa}<\delta, k \geqq \kappa$ and $h_{k} \rightarrow 0$. By Lemma 2 and the remarks immediately following it, $P\left(B_{j}, B_{k}\right) \leqq P\left(B_{j}\right) P\left(B_{k}\right)(j \neq k \geqq \kappa)$, and so by Lemma $1, P\left(B_{k}\right.$ i.o. $)=1$ if $\sum P\left(B_{k}\right)$ $=\infty$. The proof consists essentially of choosing the sequence $\left\{h_{k}\right\}$ so that $\sum P\left(B_{k}\right)$ 
diverges. Once this is shown, the remainder of the proof is simple. Each case will be treated separately.

The proof is trivial and the results uninteresting unless $\lim \inf _{h \rightarrow 0} f_{i}(h)=\infty$ $(i=1, \ldots, 3)$. Thus we shall assume that this is the case. Having made this assumption the probability of the event $B_{k}$ can be bounded below by

$$
P\left(B_{k}\right) \geqq\left(\text { Const } \frac{\sigma^{2}\left(h_{k}\right)}{\sigma^{2}\left(h_{k}-h_{k+1}\right)} f_{i}\left(h_{k}\right)\right)^{-1 / 2} \exp \left\{-C_{1, i}^{2} \frac{\sigma^{2}\left(h_{k}\right)}{\sigma^{2}\left(h_{k}-h_{k+1}\right)} f_{i}\left(h_{k}\right)\right\} \text {. }
$$

In the first case, when $f_{1}(h)=\log _{2} 1 / h$ it is easy to show that $\sum^{\infty} P\left(B_{k}\right)=\infty$. Choose $h_{k}=\theta^{k}, \theta<1$. Then $f_{1}(h)=\log k+\log _{2} 1 / \theta$ and

$$
\sigma^{2}\left(\theta^{k}\right) / \sigma^{2}\left(\theta^{k}(1-\theta)\right) \leqq 1 /(1-\theta)
$$

because of the concavity of $\sigma^{2}(h)$. For any $\varepsilon>0$ we can set $C_{1,1}=1-\varepsilon$, and by taking $\theta$ sufficiently close to zero we obtain $\sum^{\infty} P\left(B_{k}\right)=\infty$.

In Case $2, f_{2}(h)=1 / 2 g^{\prime}(\log 1 / h)$. It is only necessary to consider those processes for which $\lim \inf _{h \rightarrow 0} f_{2}(h)=\infty$. Choose $h_{k}$ as follows:

$$
h_{k}=\min \left(h \leqq \delta: 1 / 2 g^{\prime}(\log 1 / h)=\log k\right) .
$$

This is possible because by Remark 2 the only discontinuities in $1 / 2 g^{\prime}(\log 1 / h)$ as $h \rightarrow 0$ are negative jumps. Consider $\sigma^{2}\left(h_{k}\right) / \sigma^{2}\left(h_{k}-h_{k+1}\right)$. If $h_{k+1}<h_{k} / 2$, this quantity is less than 2 , as can be seen from (1.3). Assuming $h_{k+1} \geqq h_{k} / 2$, we have

$$
\begin{aligned}
\frac{\sigma^{2}\left(h_{k}\right)}{\sigma^{2}\left(h_{k}-h_{k+1}\right)} & =\exp 2\left\{g\left(\log 1 / h_{k}+\log \frac{h_{k}}{h_{k}-h_{k+1}}\right)-g\left(\log 1 / h_{k}\right)\right\} \\
& \leqq \exp 2\left\{\log \frac{h_{k}}{h_{k}-h_{k+1} \log 1 / h_{k} \leqq s \leqq \log 1 /\left(h_{k}-h_{k+1}\right)} g^{\prime}(s)\right\} \\
& \leqq \exp \left\{\log \frac{h_{k}}{h_{k}-h_{k+1}} \frac{1}{\log k}\right\}
\end{aligned}
$$

because of (1.4). Also, because of the way that the $h_{k}$ were chosen

$$
\begin{aligned}
\log k+1-\log k & =\int_{\log 1 / h_{k}}^{\log 1 / h_{k}+\log h_{k} / h_{k+1}} d\left(\frac{1}{2 g^{\prime}(s)}\right) \\
& \leqq \log \frac{h_{k}}{h_{k+1}} e \log k .
\end{aligned}
$$

This bound is determined by assuming that $1 / g^{\prime}(s)$ rises as quickly as possible over the interval of length $\log h_{k} / h_{k+1}<1$, since $h_{k} \leqq 2 h_{k+1}$ and $1 / 2 g^{\prime}\left(\log 1 / h_{k}\right)=\log k$ and $d / d s\left(1 / g^{\prime}(s)\right)<1 / g^{\prime}(s)$ as was shown in Remark 1 .

Since $\log h_{k} / h_{k+1} \leqq\left(h_{k} j h_{k+1}-1\right)$ we have

taking logarithms

$$
\frac{1}{k}\left(1-\frac{1}{2 k}\right) \leqq\left(1-\frac{h_{k+1}}{h_{k}}\right) \frac{h_{k}}{h_{k+1}} e \log k
$$

$$
\log \frac{h_{k}}{h_{k}-h_{k+1}} \leqq \log k+\text { Const } \log _{2} k
$$


Thus, for any $\varepsilon$ we can choose a $\kappa_{0}$ so that

$$
\frac{\sigma^{2}\left(h_{k}\right)}{\sigma^{2}\left(h_{k}-h_{k+1}\right)} \leqq \exp \left\{1+\frac{\text { Const } \log _{2} k}{\log k}\right\} \leqq e^{1+\varepsilon_{1}}, \quad k \geqq \kappa_{0}
$$

where the value of $\varepsilon_{1}$ is such that $C_{1,2}=(1-\varepsilon) / \sqrt{ } e \leqq\left(e^{1+\varepsilon_{1}}\right)^{-1 / 2}$. For this value of $C_{1,2}, \sum^{\infty} P\left(B_{k}\right)=\infty$.

In Case 3 we choose $h_{k}$ as follows:

$$
h_{k}=\min \left(h \leqq \delta: f_{3}(h)=\log k\right) .
$$

If $\log _{2} 1 / h_{k}=\log k$ and $\log _{2} 1 / h_{k+1}=\log k+1$, then $h_{k}=(1 / e)^{k}$ and $h_{k+1}=(1 / e)^{k+1}$ and $\sigma^{2}\left(h_{k}\right) / \sigma^{2}\left(h_{k}-h_{k+1}\right) \leqq e /(e-1)$. If $1 / 2 g^{\prime}\left(\log 1 / h_{k}\right)=\log k$ and $1 / 2 g^{\prime}\left(\log 1 / h_{k+1}\right)$ $=\log k+1$, then the analysis used in Case 2 shows that for any $\varepsilon_{1}>0$ we can choose a $\kappa_{1}$ so that $\sigma^{2}\left(h_{k}\right) / \sigma^{2}\left(h_{k}-h_{k+1}\right) \leqq e^{1+\varepsilon_{1}}$ for $k \geqq \kappa_{1}$. It should be noted, however, that because of the nature of $f_{3}(h)$, we need $g^{\prime}(s)$ to be monotonically decreasing as $s \rightarrow \infty$.

Suppose that $1 / 2 g^{\prime}\left(\log 1 / h_{k}\right)=\log k$ and $\log _{2} 1 / h_{k+1}=\log k+1$. This implies that $\log 1 / h_{k} \leqq k$ and hence that $h_{k+1} \leqq h_{k} / e$. Thus $\sigma^{2}\left(h_{k}\right) / \sigma^{2}\left(h_{k}-h_{k+1}\right) \leqq e /(e-1)$ as in (1.3).

Finally, suppose that $\log _{2} 1 / h_{k}=\log k$ and $1 / 2 g^{\prime}\left(\log 1 / h_{k+1}\right)=\log k+1$. As before, we have no difficulty if $h_{k+1}<h_{k} / 2$. If not, define $\alpha_{k}$ as follows:

$$
\alpha_{k}=\min \left(h \leqq \delta: 1 / 2 g^{\prime}(\log 1 / h)=\log k\right) \text {. }
$$

Clearly, $h_{k+1} \leqq \alpha_{k} \leqq h_{k}$. Using the concavity of $\sigma^{2}(h)$, for any $\varepsilon_{1}>0$ we can choose a $\kappa_{2}$ so that for $k \geqq \kappa_{2}$

$$
\frac{\sigma^{2}\left(h_{k}\right)}{\sigma^{2}\left(h_{k}-h_{k+1}\right)} \leqq \frac{2 \sigma^{2}\left(h_{k+1}\right)}{\sigma^{2}\left(\alpha_{k}-h_{k+1}\right)} \leqq \frac{2 \sigma^{2}\left(\alpha_{k}\right)}{\sigma^{2}\left(\alpha_{k}-h_{k+1}\right)} \leqq 2 e^{1+\varepsilon_{1}}
$$

The last inequality follows because $\alpha_{k}$ and $h_{k+1}$ are the smallest values of $h$ for which $1 / 2 g^{\prime}(\log 1 / h)$ is equal to $\log k$ and $\log k+1$ respectively. Thus, we can use the analysis of Case 2. Since $\sigma^{2}\left(h_{k}\right) / \sigma^{2}\left(h_{k}-h_{k+1}\right) \leqq 2 e^{1+\varepsilon_{1}}$, for $k$ sufficiently large, regardless of how $h_{k}$ is chosen, we obtain $\sum^{\infty} P\left(B_{k}\right)=\infty$ for $C_{1,3}$ $=(1-\varepsilon) /(2 e)^{1 / 2}$ for any $\varepsilon>0$. (The number $\varepsilon$ depends on $\varepsilon_{1}$ as in Case 2.)

In each of the cases considered we have shown that the series $\sum^{\infty} P\left(B_{k}\right)$ diverges for an appropriate choice of $C_{1, i}, i=1, \ldots, 3$. We now complete the proof of this theorem. Since $P\left(B_{k}\right.$ i.o. $)=1$, there exists a subsequence of the $h_{k}$ for which

Therefore, either

$$
\frac{X\left(t+h_{k}\right)-X\left(t+h_{k+1}\right)}{\left(2 \sigma^{2}\left(h_{k}\right) f_{i}\left(h_{k}\right)\right)^{1 / 2}} \geqq C_{1, i}
$$

$$
\frac{\left|X\left(t+h_{k}\right)-X(t)\right|}{\left(2 \sigma^{2}\left(h_{k}\right) f_{i}\left(h_{k}\right)\right)^{1 / 2}} \geqq C_{1, i} / 2
$$

or

$$
\frac{\left|X\left(t+h_{k+1}\right)-X(t)\right|}{\left(2 \sigma^{2}\left(h_{k}\right) f_{i}\left(h_{k}\right)\right)^{1 / 2}} \geqq C_{1, i} / 2 .
$$


Inequality (1.10) implies

$$
\frac{\left|X\left(t+h_{k+1}\right)-X(t)\right|}{\left(2 \sigma^{2}\left(h_{k+1}\right) f_{i}\left(h_{k+1}\right)\right)^{1 / 2}} \geqq C_{1, i} / 2\left(1-\frac{2}{k \log k}\right) .
$$

Since either (1.9) or (1.11) holds infinitely often, the theorem is proved. The constants $C_{i}$ are effectively $C_{1, i} / 2$ since the $\varepsilon$ 's can be altered to accommodate the last term in (1.11).

We now turn to the lower bound for the uniform Hölder condition. This is much easier to obtain than the Hölder condition at a point and presents no surprises since the results are the same as for the Brownian motion.

THEOREM 2. Let $X(t)$ be a separable, real-valued Gaussian process with stationary increments for which $E\left\{(X(t+h)-X(t))^{2}\right\}=\sigma^{2}(h)$ where $\sigma^{2}(h)$ is concave for $h \in[0, \delta]$ for some $\delta>0, \sigma^{2}(h) \rightarrow 0$ as $h \rightarrow 0$. Then

$$
P\left\{\limsup _{\left|t-t^{\prime}\right|=h \rightarrow 0 ; 0 \leqq t, t^{\prime} \leqq 1} \frac{\left|X(t)-X\left(t^{\prime}\right)\right|}{\left(\sigma^{2}(h)\left(2 \log 1 / h+c \log _{2} 1 / h\right)\right)^{1 / 2}}>1\right\}=1
$$

where $c<-1$ (see [5]).

Proof. It will suffice if we show that

$$
P\left\{\lim _{n \rightarrow \infty ; 1 \leqq j \leqq n} \frac{|X[(j / n) \delta]-X[((j-1) / n) \delta]|}{\left(\sigma^{2}(\delta / n)\left(2 \log n / \delta+c \log _{2} n / \delta\right)\right)^{1 / 2}}>1\right\}=1 .
$$

Notice that we restrict ourselves to the region where the covariance is convex. Define

$$
B_{j, n}=\left\{X \mid X\left(\frac{j}{n} \delta\right)-X\left(\frac{j-1}{n} \delta\right)>\left(\sigma^{2}(\delta / n)\left(2 \log n / \delta+c \log _{2} n / \delta\right)\right)^{1 / 2}\right\}
$$

Since the process has stationary increments, the sets $B_{j, n}$ have the same probability measure for $j=1,2, \ldots, n$. $P\left(B_{j, n}, B_{k, n}\right) \leqq P\left(B_{j, n}\right) P\left(B_{k, n}\right)$ because of the concavity of $\sigma^{2}(h)$. Thus we can use Corollary 1 which says that the event $B_{n}=\bigcup_{j=1}^{n} B_{j, n}$ is realized infinitely often with probability 1 if $\lim _{n \rightarrow \infty} n P\left(B_{j, n}\right)=\infty$. This limit is infinite since for $n$ sufficiently large

$$
n P\left(B_{j, n}\right) \geqq \frac{\text { Const }}{\left(2 \log n / \delta+c \log _{2} n / \delta\right)^{1 / 2}} \exp \left\{\log n-\left(\log n / \delta+(c / 2) \log _{2} n / \delta\right)\right\}
$$

and $c<-1$ while $\delta$ remains fixed. The fact that $P\left(B_{n}\right.$ i.o. $)=1$ provides a proof of this theorem. Actually we know more because Corollary 1 asserts that $\lim _{\inf _{n \rightarrow \infty}} P\left(B_{n}\right)=1$.

An immediate corollary of this theorem is Belyaev's [6] result on a sufficient condition for a Gaussian process with stationary increments to have discontinuous sample paths. (Belyaev obtained the result for stationary processes; however, the extension is trivial.)

COROLlARY 2. Let $X(t)$ be a separable, real-valued Gaussian process with 
stationary increments for which $E\left\{(X(t+h)-X(t))^{2}\right\}=\sigma^{2}(h)$, where $\sigma^{2}(h)$ is concave for $h \in[0, \delta]$ for some $\delta>0, \sigma^{2}(0)=0$. Then a sufficient condition that almost all sample paths of $X(t)$ are discontinuous in all intervals is that $\sigma^{2}(h) \geqq C /|\log | h||$ for some $C>0$.

We end this section by mentioning that the work of the author in [7] can be used to extend the results of Theorem 2 to a larger class of processes than those for which $\sigma^{2}(h)$ is concave.

2. Upper bounds. Upper bounds, first for the almost sure Hölder conditions at a point and then for the almost sure uniform Hölder conditions for continuous, separable, real-valued Gaussian processes with stationary increments, will be determined.

The major tool used in this section is the following lemma due to X. Fernique [3].

LemMA 4. Let $Y(t)$ be a continuous, separable, real-valued Gaussian process on $[0,1]$ with zero mean and continuous covariance $\Gamma(t, s)$. Suppose that

$$
E\left\{(Y(t)-Y(s))^{2}\right\} \leqq \sigma^{2}(|t-s|)
$$

and that $\sigma(h)$ is positive and increasing in $h$ for $h \geqq 0$. Then for all positive integers $n$ and all $x \geqq(1+4 \log n)^{1 / 2}$, we have

$$
P\left\{\|Y\|_{\infty} \geqq x\left(\left(\|\Gamma\|_{\infty}\right)^{1 / 2}+4 \int_{1}^{\infty} \sigma\left(n^{-u^{2}}\right) d u\right)\right\} \leqq 4 n^{2} \int_{x}^{\infty} e^{-u^{2} / 2} d u
$$

\|\|$_{\infty}$ is the sup norm.

We proceed to Theorem 3.

THEOREM 3. Let $X(t)$ be a separable, real-valued Gaussian process with stationary increments. Let $E\left\{(X(t)-X(s))^{2}\right\}=\sigma^{2}(|t-s|)$ where $\sigma(h)$ is positive and increasing in $h, h \geqq 0$. Assume further that $\int^{\infty} \sigma\left(e^{-x^{2}}\right) d x$ converges. Then

$$
P\left\{\limsup _{h \rightarrow 0} \frac{|X(t+h)-X(t)|}{\left(2 \sigma^{2}(h) f_{i}(h)\right)^{1 / 2}}<C\right\}=1,
$$

$i=1, \ldots, 3, C=(1+\varepsilon)(12)^{1 / 2}$ and $f_{i}(h)$ can be any of the following functions. (The processes are subjected to whatever additional conditions are listed.)

Case 1.

$$
f_{1}(h)=\max \left(\log _{2} 1 / h, \max _{h \leqq t \leqq 1} \frac{2}{\sigma(t)} \int_{0}^{t} \frac{\sigma(u)}{u} d u\right)
$$

as long as

$$
\int_{0}^{t} \frac{\sigma(u)}{u} d u<\infty
$$

Case 2.

$$
f_{2}(h)=\max \left(\log _{2} 1 / h, \frac{2}{\sigma(h)} \int_{0}^{h} \frac{\sigma(u)}{u} d u\right)
$$


if $\sigma(h)$ is concave for $h \in[0, \delta]$ for some $\delta>0$ and

$$
\int_{0}^{h} \frac{\sigma(u)}{u} d u<\infty
$$

Case 3.

$$
f_{3}(h)=\max \left(\log 1 / h, \max _{h \leqq t \leq 1}\left\{\frac{4 \sqrt{ } 2}{\sigma(t)} \int_{(2 \log 1 / t)^{1 / 2}}^{\infty} \sigma\left(e^{-x^{2}}\right) d x\right\}^{2}\right) .
$$

Proof. Note that the convergence of $\int^{\infty} \sigma\left(e^{-x^{2}}\right) d x$ is a sufficient condition that the associated Gaussian process has continuous sample paths [3]. In Cases 1 and 2, it is required that $\int_{0}^{t}(\sigma(u) / u) d u=2 \int_{(\log 1 / t)^{1 / 2}}^{\infty} x \sigma\left(e^{-x^{2}}\right) d x$ converges. Thus, the Gaussian processes for which these integrals converge also have continuous sample paths. Cases 1 and 2 provide the more interesting results since for many processes $\log _{2} 1 / t \leqq(2 / \sigma(t)) \int_{0}^{t}(\sigma(u) / u) d u \leqq \log 1 / t$. Furthermore, for many values of $\sigma(t),(1 / \sigma(t)) \int_{0}^{t}(\sigma(u) / u) d u$ is asymptotic to $1 / g^{\prime}(\log 1 / t)$, the function that appeared in the results for the lower bound.

We begin the proof by rewriting Fernique's lemma so that the supremum is taken over $\left[0, t_{k}\right]$ rather than $[0,1]$. Define

$$
Y(t)=X\left(t t_{k}+t_{0}\right)-X\left(t_{0}\right), \quad 0 \leqq t \leqq 1
$$

Then $Y(t)$ is a continuous Gaussian process on $[0,1]$

$$
E\left\{|Y(t)-Y(s)|^{2}\right\} \leqq \sigma^{2}\left(|t-s| t_{k}\right)
$$

and

$$
\begin{aligned}
E\{Y(t) Y(s)\} & =\Gamma(t, s) \leqq\left[E\left\{Y(t)^{2}\right\} E\left\{Y(s)^{2}\right\}\right]^{1 / 2} \\
& \leqq\left\{\sigma^{2}\left(t t_{k}\right) \sigma^{2}\left(s t_{k}\right)\right\}^{1 / 2} \leqq \sigma^{2}\left(t_{k}\right), \quad 0 \leqq s, t \leqq 1
\end{aligned}
$$

Define the set of paths $B_{k}$ as follows:

$$
B_{k}=\left\{X\left|\sup _{0 \leqq t \geqq t_{k}}\right| X\left(t+t_{0}\right)-X\left(t_{0}\right) \mid \geqq C_{1}\left(2 \sigma^{2}\left(t_{k}\right) f_{i}\left(t_{k}\right)\right)^{1 / 2}\right\}
$$

By Lemma 4

$$
P\left(B_{k}\right) \leqq 4 n^{2} \int_{y}^{\infty} e^{-u^{2} / 2} d u
$$

where

$$
y=C_{1}\left(2 f_{i}\left(t_{k}\right)\right)^{1 / 2}\left\{1+\frac{4}{\sigma\left(t_{k}\right)} \int_{1}^{\infty} \sigma\left(n^{-u^{2}} t_{k}\right) d u\right\}^{-1}
$$

providing that $y$ is greater than $(1+4 \log n)^{1 / 2}$. As we shall see, $y \rightarrow \infty$ as $t_{k} \rightarrow 0$. Thus

$$
P\left(B_{k}\right) \leqq 4 \exp \left\{2 \log n-\frac{C_{1}^{2} f_{i}\left(t_{k}\right)}{\left(1+4 / \sigma\left(t_{k}\right) \int_{1}^{\infty} \sigma\left(n^{-u^{2}}\right) d u\right)^{2}}\right\}
$$


Our object is to show that $\sum P\left(B_{k}\right)<\infty$ (and then to use the Borel-Cantelli lemma). To do this we choose a sequence $t_{k} \rightarrow 0$, and then for each $t_{k}$ we choose $n$ so that either $\log n$ and $f\left(t_{k}\right)$ are approximately equal, or $f\left(t_{k}\right)$ is greater than $\log n$. We show that for this choice of $\log n$ the denominator on the right in (2.3) can be bounded above by a constant, uniformly for all $t_{k}$. Then by choosing $C_{1}$ large enough the term on the right in (2.3) will dominate the $2 \log n$ term. Finally, we show that $\sum^{\infty} P\left(B_{k}\right)$ converges.

We will now prove Case 1 . The sequence $t_{k}$ is chosen as follows: starting with some value $t_{0}$, which may be as small as we wish, define $t_{k}$ by the equation $\sigma\left(t_{k}\right)$ $=\theta \sigma\left(t_{k-1}\right), \theta<1$. This is possible since $\sigma(h) \rightarrow 0$ as $h \rightarrow 0$. For a given value of $t_{k}$ define $n$ as follows:

$$
n=\left[\exp \left\{\frac{2}{\sigma\left(t_{k}\right)} \int_{0}^{t_{k}} \frac{\sigma(u)}{u} d u\right\}\right]+1
$$

where [ ] indicates integral part. Note that $\log x \leqq \log ([x]+1) \leqq \log x+1, x \geqq 1$. Thus

$$
\frac{2}{\sigma\left(t_{k}\right)} \int_{0}^{t_{k}} \frac{\sigma(u)}{u} d u \leqq \log n \leqq \frac{2}{\sigma\left(t_{k}\right)} \int_{0}^{t_{k}} \frac{\sigma(u)}{u} d u+1
$$

Since

$$
\begin{aligned}
\frac{4}{\sigma\left(t_{k}\right)} \int_{1}^{\infty} \sigma\left(n^{-u^{2}} t_{k}\right) d u & =\frac{2}{\sigma\left(t_{k}\right)(\log n)^{1 / 2}} \int_{0}^{t_{k} / n} \frac{\sigma(v) d v}{v\left(\log 1 / v-\log 1 / t_{k}\right)^{1 / 2}} \\
& \leqq \frac{2}{\sigma\left(t_{k}\right) \log n} \int_{0}^{t_{k} / n} \frac{\sigma(v)}{v} d v,
\end{aligned}
$$

we see that the last term in (2.6) is bounded above by 1 . If we choose $C_{1} \geqq(1+\varepsilon)(12)^{1 / 2}$ for some $\varepsilon>0$, we obtain

$$
P\left(B_{k}\right) \leqq \text { Const } \exp \left(-(1+\varepsilon) f_{1}\left(t_{k}\right)\right) \leqq \exp \left(-(1+\varepsilon) \log _{2} 1 / t_{k}\right) .
$$

Note that $y$ as defined in (2.2) is greater than $(1+4 \log n)^{1 / 2}$.

Recall that the function $\sigma^{2}(h)$ is such that $E\left\{(X(t+h)-X(t))^{2}\right\}=\sigma^{2}(h)$, where $X(t)$ is a real-valued Gaussian process with stationary increments for which (1.1) holds. Since $\sigma(h)>0$ for $h>0$, there exists a $\delta>0$ such that for $h \in[0, \delta], \sigma(h)>h^{1+n}$ for any $\eta>0$. The proof of this fact is the same as in the stationary case. Therefore $t_{k}^{1+\eta}<\sigma\left(t_{k}\right)=\theta^{\kappa} \sigma\left(t_{0}\right)$. Using this inequality in (2.7) we see that $\sum^{\infty} P\left(B_{k}\right)$ converges.

It is significant to note that this is the only place in which it is required that $\sigma^{2}(h)$ be the incremental covariance of a Gaussian process with stationary increments. Fernique's lemma requires only that $E\left\{(X(t+h)-X(t))^{2}\right\} \leqq \sigma^{2}(h)$ where $\sigma(h)$ is increasing in $h \geqq 0$. Therefore, we can replace the equality in the statement of this theorem with the inequality for $\sigma(h)$ increasing as $h \geqq 0$ increases if we add the condition that $\sigma(t)>t^{1+n}$ for $t$ in some [0, $\left.\delta\right]$ interval. This would be the case if $\sigma(h)$ is concave, or if it is the incremental covariance of some other Gaussian process with stationary increments. 
By the Borel-Cantelli lemma, we know that except for a set of paths of measure zero, to each path $X$ we can associate an integer $K(X)$ such that for all $k \geqq K(X)$,

$$
\sup _{0 \leqq h \leqq t_{k}} \frac{|X(t+h)-X(t)|}{\left(2 \sigma^{2}\left(t_{k}\right) f_{1}\left(t_{k}\right)\right)^{1 / 2}}<C_{1} .
$$

Thus

$$
\sup _{t_{k+1} \leqq h \leqq t_{k}} \frac{|X(t+h)-X(t)|}{\left(2 \sigma^{2}\left(t_{k+1}\right) f_{1}\left(t_{k}\right)\right)^{1 / 2}}<\frac{1}{\theta} C_{1}
$$

Since $f_{1}(h)$ increases monotonically as $h \rightarrow 0$,

$$
\sup _{t_{k+1} \leqq h \leqq t_{k}} \frac{|X(t+h)-X(t)|}{\left(2 \sigma^{2}(h) f_{1}(h)\right)}<\frac{1}{\theta} C_{1} .
$$

Since (2.9) holds for all $k \geqq K(X)$, the theorem is proved for Case 1. Taking $\theta$ close to 1 and choosing some new value of $\varepsilon>0$, we obtain $C \geqq(1+\varepsilon)(12)^{1 / 2}$ for this case.

In proving Case 2 , we utilize the concavity of $\sigma^{2}(h)$. The sequence $t_{k}$ is defined by $t_{k}=\theta^{k} t_{0}$ where $\theta<1$ and $t_{0}$ may be taken as small as we wish. Notice that the proof in this case is identical to the proof of Case 1 up to (2.8). The choice of $n$, which is the key step, depends on $t_{k}$ but it does not matter how $t_{k}$ is chosen. In order to proceed from (2.8) to (2.9) for $f_{2}(h)$ it suffices to show that

$$
\min _{t_{k+1} \leqq h \leqq t_{k}} \sigma^{2}(h) f_{2}(h)>\theta \sigma^{2}\left(t_{k}\right) f_{2}\left(t_{k}\right)
$$

and to show this all we need to do is to obtain (2.10) when $f_{2}(h)=\log _{2} 1 / h$ and when $f_{2}(h)=(1 / \sigma(h)) \int_{0}^{h}(\sigma(u) / u) d u$. Those cases when both $\log _{2} 1 / h$ and

$$
(1 / \sigma(h)) \int_{0}^{h}(\sigma(u) / u) d u
$$

contribute to $f_{2}(h)$ for $t_{k+1} \leqq h \leqq t_{k}$ are encompassed by these two cases. We see that

$$
\min _{t_{k+1} \leqq h \leqq t_{k}} \sigma^{2}(h) \log _{2} 1 / h \geqq \sigma^{2}\left(t_{k+1}\right) \log _{2} 1 / t_{k} \geqq \theta \sigma^{2}\left(t_{k}\right) \log _{2} 1 / t_{k}
$$

since $\sigma^{2}\left(\theta t_{k}\right) \geqq \theta \sigma^{2}\left(t_{k}\right)$. Also

$$
\begin{aligned}
\min _{t_{k+1} \leqq h \leqq t_{k}} \sigma(h) \int_{0}^{h} \frac{\sigma(u)}{u} d u & =\sigma\left(t_{k+1}\right) \int_{0}^{t_{k+1}} \frac{\sigma(u)}{u} d u \\
& \geqq \sqrt{ } \theta \sigma^{2}\left(t_{k}\right)\left[\frac{1}{\sigma\left(t_{k}\right)} \int_{0}^{t_{k}} \frac{\sigma(u)}{u} d u-\frac{1}{\sigma\left(t_{k}\right)} \int_{t_{k+1}}^{t_{k}} \frac{\sigma(u)}{u} d u\right] .
\end{aligned}
$$

The last term in the brackets in (2.11) can be bounded above by $(1-\theta) / \theta$. Since the first term $\rightarrow \infty$ as $t_{k} \rightarrow 0$ we obtain (2.10). Thus, the theorem is proved in Case 2. The constant $C$ remains the same. 
For Case 3, starting with some small value of $t_{0}$ define $t_{k}=\theta \sigma\left(t_{k-1}\right), \theta<1$. For a fixed value of $t_{k}$ set $n=\left[1 / t_{k}\right]+1$. Then $\log 1 / t_{k} \leqq \log n \leqq \log 1 / t_{k}+1$. Notice that

$$
\frac{4}{\sigma\left(t_{k}\right)} \int_{1}^{\infty} \sigma\left(n^{-u^{2}} t_{k}\right) d u \leqq \frac{4 \sqrt{ } 2}{\left(\log 1 / t_{k}\right)^{1 / 2} \sigma\left(t_{k}\right)} \int_{\left(2 \log 1 / t_{k}\right)^{1 / 2}}^{\infty} \sigma\left(e^{-x^{2}}\right) d x .
$$

Therefore, analogous to (2.3)

$$
P\left(B_{k}\right) \leqq \text { Const } \exp \left[2 \log 1 / t_{k}-\frac{C_{1}^{2} f_{3}^{2}\left(t_{k}\right) \log 1 / t_{k}}{\left\{\left(\log 1 / t_{k}\right)^{1 / 2}+\frac{4 \sqrt{ } 2}{\sigma\left(t_{k}\right)} \int_{\left(2 \log 1 / t_{k}\right)^{1 / 2}}^{\infty} \sigma\left(e^{-x^{2}}\right) d x\right\}^{2}}\right]
$$

Thus, we can obtain $P\left(B_{k}\right) \leqq$ Const $\exp \left\{-(1+\varepsilon) \log 1 / t_{k}\right\}$ for any $\varepsilon>0$, and $\sum^{\infty} P\left(B_{k}\right)<\infty$. Since $f_{3}(h)$ increases monotonically, the remainder of the proof of this case is identical to the proof of Case 1. This finishes the proof of the theorem.

We now turn our attention to uniform Hölder conditions. The proof of the next theorem follows the proof of P. Levy's Hölder condition for the Brownian motion which appears in his book L'addition des variables aléatoires, [8, p. 164]. However, at two points the increased generality of the class of functions $\sigma^{2}(h)$ requires additional work. In the first case, Fernique's lemma is used and in the second, we impose an asymptotic bound on $1 / g^{\prime}(\log 1 / h)$.

THEOREM 4. Let $X(t)$ be a real-valued, continuous, separable Gaussian process. Assume that $E\left\{(X(t+h)-X(t))^{2}\right\} \leqq \sigma^{2}(h) ; \sigma^{2}(h)$ is assumed to be concave in $[0, \delta]$ for some $\delta>0$. Assume also that $1 / g^{\prime}(\log 1 / h)=o(\log 1 / h)$. Then

$$
P\left\{\limsup _{\left|t-t^{\prime}\right|=h \rightarrow 0 ; 0 \leqq t, t^{\prime} \leqq 1} \frac{\left|X(t)-X\left(t^{\prime}\right)\right|}{\left(2 \sigma^{2}(h) \log 1 / h\right)^{1 / 2}} \leqq 1\right\}=1
$$

for any $\varepsilon>0$.

Proof. As a first step we use the Borel-Cantelli lemma to show that, except for a set of paths of measure zero, for each path $X$ there exists an integer $P$ so that for all $p \geqq P$,

$$
\left|X\left(q / 2^{p}+t\right)-X\left(q / 2^{p}\right)\right|<C_{1}\left(2 \sigma^{2}(t) \log 1 / t\right)^{1 / 2}
$$

for $\left|t-q / 2^{p}\right| \leqq 2^{-p}$ and all $q=0,1,2, \ldots, 2^{p-1}$, with $C_{1}=1+\varepsilon / 2, \varepsilon>0$. Define the following set of paths:

$$
\begin{aligned}
& B_{p}=\{X \mid \text { For at least one } q \in Q(p), k \in J ; \\
& \left.\sup _{0 \leqq t \leqq t_{k}}\left|X\left(\frac{q}{2^{p}}+t\right)-X\left(\frac{q}{2^{p}}\right)\right| \geqq C_{2}\left(2 \sigma^{2}\left(t_{k}\right) \log 1 / t_{k}\right)^{1 / 2}\right\}
\end{aligned}
$$

where $Q(p)=1,2, \ldots, 2^{p}-1, J$ is the positive integers including $0, C_{2}=1+\varepsilon / 4$ and $t_{k}=2^{-p} \theta^{k}, \theta<1$.

Let us choose a $t^{\prime}$ sufficiently small so that $1 / g^{\prime}(\log 1 / t)<\alpha \log 1 / t$ for $t<t^{\prime}$, where $\alpha$ is a small positive number. 
We shall see that $B_{p}$ is dependent on $\sigma(t)$ only for values of $t \leqq 2^{-p}$. Thus let us choose an integer $P_{0}$ so that for $p \geqq P_{0}, 2^{-p}<t^{\prime}$. We shall restrict ourselves to sets $B_{p}$ for $p \geqq P_{0}$, that is, to sets which depend on $\sigma^{2}(t)$ for values of $t$ for which $1 / g^{\prime}(\log 1 / t)<\alpha \log 1 / t$.

$$
P\left\{B_{p}\right\} \leqq 2^{p} \sum_{k=0}^{\infty} P\left\{X\left|\sup _{0 \leqq t \leqq t_{k}}\right| X(t)-X(0) \mid \geqq C_{2}\left(2 \sigma^{2}\left(t_{k}\right) \log 1 / t_{k}\right)^{1 / 2}\right\}
$$

From (2.1) we see that

$$
P\left\{X\left|\sup _{0 \leqq t \geqq t_{k}}\right| X(t)-X(0) \mid \geqq C_{2}\left(2 \sigma^{2}\left(t_{k}\right) \log 1 / t_{k}\right)^{1 / 2}\right\} \leqq 4 n^{2} \int_{y}^{\infty} e^{-u^{2} / 2} d u
$$

where

$$
y=C_{2}\left(2 \log 1 / t_{k}\right)^{1 / 2} /\left(1+\frac{4}{\sigma\left(t_{k}\right)} \int_{1}^{\infty} \sigma\left(n^{-u^{2}} t_{k}\right) d u\right)
$$

and $n$ is an integer greater than zero such that $\log n<2^{-1} \log 1 / t_{k}$. Inequality (2.14) is strengthened if we replace $y$ by (see Eq. 2.6)

$$
y^{\prime}=C_{2}\left(\log 1 / t_{k}\right)^{1 / 2} /\left(1+\frac{2}{\sigma\left(t_{k}\right) \log n} \int_{0}^{t_{k}} \frac{\sigma(u)}{u} d u\right) .
$$

Note that $t_{k} \leqq t_{0}=2^{-p}$. Thus by our previous remarks we can assume that $1 / g^{\prime}(\log 1 / t)<\alpha \log 1 / t$. We have

$$
\begin{aligned}
\frac{1}{\sigma\left(t_{k}\right)} \int_{0}^{t_{k}} \frac{\sigma(u)}{u} d u & =\frac{1}{\sigma\left(t_{k}\right)} \int_{0}^{t_{k}} \frac{\sigma(u)}{u \sigma^{\prime}(u)} d \sigma(u) \\
& \leqq \frac{1}{\sigma\left(t_{k}\right)} \int_{0}^{t_{k}} \alpha \log \frac{1}{u} d \sigma(u)=\alpha \log \frac{1}{t_{k}}+\frac{\alpha}{\sigma\left(t_{k}\right)} \int_{0}^{t_{k}} \frac{\sigma(u)}{u} d u .
\end{aligned}
$$

Therefore,

$$
\frac{1}{\sigma\left(t_{k}\right)} \int_{0}^{t_{k}} \frac{\sigma(u)}{u} d u \leqq \frac{\alpha}{1-\alpha} \log \frac{1}{t_{k}}
$$

Let $n=\left[\left(1 / t_{k}\right)^{\sqrt{ } \alpha}\right]+1$, then $\sqrt{ } \alpha \log 1 / t_{k} \leqq \log n \leqq \sqrt{ } \alpha \log 1 / t_{k}+1$. We then obtain as an upper bound for (2.14)

$$
4 \exp \left\{2 \sqrt{ } \alpha\left(\log 1 / t_{k}+1\right)-\frac{C_{2}^{2} \log 1 / t_{k}}{[1+(2 \sqrt{ } \alpha / 1-\alpha)]^{2}}\right\} \text {. }
$$

Since $C_{2}=1+\varepsilon / 4$, we see that for $\alpha$ small enough, (2.17) can be bounded above by $\exp \left\{-c^{2} \log 1 / t_{k}\right\}$ where $c=1+\varepsilon^{\prime}$ for some sufficiently small $\varepsilon^{\prime}>0$. Therefore, since $t_{k}=2^{-p} \theta^{k}$,

$$
P\left\{B_{p}\right\}=2^{p} \sum_{k=0}^{\infty} \exp \left\{-c^{2} \log 1 / t_{k}\right\}=\frac{1}{2^{p\left(c^{2}-1\right)}} \sum_{k=0}^{\infty}\left(\theta^{c^{2}}\right)^{k} \leqq \text { Const } \frac{1}{2^{p\left(c^{2}-1\right)}} .
$$

Since $\Sigma^{\infty} P\left(B_{p}\right)<\infty$, by the Borel-Cantelli lemma $P\left\{B_{p}\right.$ infinitely often $\}=0$. 
We have shown that with the exception of a set of paths of probability zero, for each path $X$ there exists an integer $P$, so that for $p \geqq P$ the following inequality is satisfied for all $q \in Q(p)$ and $k \in J$ :

$$
\sup _{0 \leqq t \leqq t_{k}}\left|X\left(q / 2^{p}+t\right)-X\left(q / 2^{p}\right)\right|<C_{1} \theta\left(2 \sigma^{2}\left(t_{k}\right) \log 1 / t_{k}\right)^{1 / 2}
$$

where we choose $\theta$ large enough so that $C_{1} \theta>C_{2}$. Notice that since $\sigma(t)$ is concave, $\sigma(a t)>a \sigma(t)$ for $0 \leqq a \leqq 1$. Thus $\sigma\left(t_{k+1}\right)=\sigma\left(2^{-p} \theta^{k+1}\right) \geqq \theta \sigma\left(2^{-p} \theta^{k}\right)=\theta \sigma\left(t_{k}\right)$, and

$$
\sup _{0 \leqq t \leqq t_{k}}\left|X\left(\frac{q}{2^{p}}+t\right)-X\left(\frac{q}{2^{p}}\right)\right|<C_{1}\left(2 \sigma^{2}\left(t_{k+1}\right) \log 1 / t_{k}\right) .
$$

Therefore

$$
\left|X\left(\frac{q}{2^{p}}+t\right)-X\left(\frac{q}{2^{p}}\right)\right|<C_{1}\left(2 \sigma^{2}(t) \log \frac{1}{t}\right)^{1 / 2}, \quad t_{k+1} \leqq t \leqq t_{k} .
$$

Since this holds for all $k$ we have the desired result (Eq. (2.12)) if the supremum is taken over $t-q / 2^{p}>0$. However, we can repeat the proof for $t-q / 2^{p}$ negative, thus establishing (2.12).

The second step of this proof consists of another application of the BorelCantelli lemma. Given a small number $b>0$, we show that, with the exception of a set of paths of measure zero, for each path $X$ there exists an integer $P_{1}$ such that for all $p \geqq P_{1}$ and for all $q \in Q(p)$ and $\nu=1,2, \ldots,\left[2^{p b}\right]$ :

$$
\left|X\left(\frac{q+\nu}{2^{p}}\right)-X\left(\frac{q}{2^{p}}\right)\right|<C_{3}\left(2 \sigma^{2}\left(\frac{\nu}{2^{p}}\right) \log \frac{2^{p}}{\nu}\right)^{1 / 2}
$$

where $C_{3}=1+\varepsilon / 2$.

The probability of the reverse of the inequality in (2.19) for at least one $q \in Q(p)$, and one value of $\nu=1,2, \ldots,\left[2^{p b}\right]$ is bounded above by (for ease of notation set $C_{3}=c$ ),

$$
2^{p} \sum_{\nu=1}^{\left[2^{p b]}\right.} \frac{\nu^{c^{2}}}{2^{p c^{2}}}<\left(2^{p b}\right)^{1+c^{2}} \frac{1}{2^{p\left(c^{2}-1\right)}}=\frac{1}{2^{p\left(c^{2}(1-b)-(1+b)\right)}}
$$

For $b=\varepsilon / 2$ this is a term of a convergent series in $p$ and thus the second step is completed.

We are now ready to complete the theorem by extending (2.12) from the rational numbers to all pairs of real numbers $t$ and $t^{\prime}$ providing that they are sufficiently close. Again we make use of the bound on the asymptotic behavior of $1 / g^{\prime}(\log 1 / t)$ by choosing a $t^{\prime \prime}$ so that for $t \leqq t^{\prime \prime}, 1 / g^{\prime}(\log 1 / t)<\beta \log 1 / t$ for a small value of $\beta$ which we shall determine below. Once this value of $\beta$ is determined we choose a $P_{2}$ so that for $p \geqq P_{2}, 2^{-p(1-b)}<t^{\prime \prime}$.

Notice that for a given path $X$ (again excepting a set of measure zero) there are many conditions on the lower bound of the integers $p$, imposed both by the bounds on $1 / g^{\prime}(\log 1 / t)$ and by the applications of the Borel-Cantelli lemmas. Nevertheless, 
for each $X$ we can find a sufficiently large integer $P^{\prime}$ so that for $p \geqq P^{\prime}$ all the conditions are satisfied. Suppose that we have selected such a path $X$ and the corresponding $P^{\prime}$. We then choose a $t^{\prime}$ and $t$ (where without loss of generality we may assume that $t^{\prime}>t$ ) such that the following inequality is satisfied, $p \geqq P^{\prime}$ :

$$
\left[2^{p b}\right] / 2^{p+1}<t^{\prime}-t \leqq\left[2^{p b}\right] / 2^{p} .
$$

Determine $q, q^{\prime}, t_{1}, t_{1}^{\prime}$ by the conditions

$$
\frac{q}{2^{p}}<t \leqq t_{1}=\frac{q+1}{2^{p}}<t_{1}^{\prime}=\frac{q^{\prime}}{2^{p}} \leqq t^{\prime}<\frac{q^{\prime}+1}{2^{p}} .
$$

We write

$$
\left|X\left(t^{\prime}\right)-X(t)\right| \leqq\left|X\left(t^{\prime}\right)-X\left(t_{1}^{\prime}\right)\right|+\left|X\left(t_{1}^{\prime}\right)-X\left(t_{1}\right)\right|+\left|X\left(t_{1}\right)-X(t)\right| .
$$

Since $q^{\prime}-(q+1) \leqq\left[2^{p b}\right] / 2^{p}$ we obtain from (2.19) that the second term on the right in $(2.22)$ is bounded by

$$
(1+\varepsilon / 2)\left(2 \sigma^{2}\left(\frac{q^{\prime}-(q+1)}{2^{p}}\right) \log \frac{2^{p}}{q^{\prime}-q+1}\right)^{1 / 2}
$$

with probability 1 . From (2.12) we see that the first and third terms are bounded above by

$$
(1+\varepsilon / 2)\left(2 \sigma^{2}\left(2^{-p}\right) \log 2^{p}\right)^{1 / 2} .
$$

Here we make use of the fact that $1 / g^{\prime}(\log 1 / h) \leqq 2 \log 1 / h$ implies that $\sigma^{2}(h) \log 1 / h$ increases monotonically as $h$ increases. Finally we show that

$$
(1+\varepsilon / 2)\left(2 \sigma^{2}\left(1 / 2^{p}\right) \log 2^{p}\right)^{1 / 2}<\varepsilon / 8\left(2 \sigma^{2}\left(\frac{q^{\prime}-(q+1)}{2^{p}}\right) \log \frac{2^{p}}{q^{\prime}-(q+1)}\right)^{1 / 2}
$$

From (2.20) and (2.21) we see that

$$
2^{p b} / 4 \leqq q^{\prime}-(q+1) \leqq 2^{p b} .
$$

Thus

$$
\begin{aligned}
\sigma^{2}\left(1 / 2^{p}\right) \log 2^{p} & =\sigma^{2}\left(1 / 2^{p}\right)\left[\log \frac{2^{p}}{q^{\prime}-(q+1)}+\log \left(q^{\prime}-(q+1)\right)\right] \\
& \leqq 2 \sigma^{2}\left(1 / 2^{p}\right) \log \frac{2^{p}}{q^{\prime}-(q+1)},
\end{aligned}
$$

because by (2.26) $2^{p / 2}>q^{\prime}-(q+1)$. (Keep in mind that $b$ is taken to be very small and $p$ large.) Next we assert that $\sigma^{2}\left(1 / 2^{p}\right)<\varepsilon / 20 \sigma^{2}\left(\left(q^{\prime}-(q+1)\right) / 2^{p}\right)$. Since

$$
\left(q^{\prime}-(q+1)\right) / 2^{p} \leqq 1 / 4 \cdot 2^{p(1-b)},
$$

it suffices to show that

$$
\frac{\sigma\left(1 / 2^{p}\right)}{\sigma\left(\frac{1}{4}\left(1 / 2^{p(1-b)}\right)\right)}<\frac{4 \sigma\left(1 / 2^{p}\right)}{\sigma\left(1 / 2^{p(1-b)}\right)}<\varepsilon / 20 .
$$


We have

$$
\log \frac{\sigma\left(1 / 2^{p}\right)}{\sigma\left(1 / 2^{p(1-b)}\right)}=-\int_{1 / 2^{p}}^{1 / 2^{p(1-b)}} \frac{\sigma^{\prime}(u)}{\sigma(u)} d u-\int_{1 / 2^{p}}^{1 / 2^{p(1-b)}} \frac{d u}{\beta u \log 1 / u}=1 / \beta(\log (1-b))
$$

because $1 / g^{\prime}(\log 1 / u)=\sigma(u) / u \sigma^{\prime}(u) \leqq \beta \log 1 / u$. Thus

$$
\frac{\sigma\left(1 / 2^{p}\right)}{\sigma\left(1 / 2^{p(1-b)}\right)} \leqq(1-b)^{1 / \beta}
$$

This can be made to satisfy (2.27) for $\beta$ sufficiently small.

By the monotonicity of $\sigma^{2}(h) \log 1 / h$ we see that (2.22) is bounded by

$$
(1+\varepsilon)\left(2 \sigma^{2}(h) \log 1 / h\right)^{1 / 2} ; \quad h=t^{\prime}-t
$$

with probability 1 . Since this bound holds for all $\varepsilon>0$ the theorem is proved.

The requirement that $1 / g^{\prime}(\log 1 / t)=o(\log 1 / t)$ was used so that the constant in the theorem could be taken to be 1 . We could relax the condition on $1 / g^{\prime}(\log 1 / t)$ to make it less than $\beta \log 1 / t$, for some $\beta<1$, and still obtain the results of the theorem except that the constant could be quite large. (It is a function of $(1-\beta)^{-1}$.) The requirement that $\beta$ must be less than 1 is imposed in (2.16).

3. Relations between upper and lower Hölder conditions. In the previous sections, upper and lower bounds were obtained for the Hölder conditions of Gaussian processes with stationary increments. In this section we shall single out those processes for which the bounds differ by a constant. That is, if we set

$$
E\left\{(X(t+h)-X(t))^{2}\right\}=\sigma^{2}(h),
$$

we seek those processes for which the Hölder conditions can be expressed in the following form:

$$
C_{0} \leqq \limsup _{h \rightarrow 0} \frac{|X(t+h)-X(t)|}{\left(2 \sigma^{2}(h) f(h)\right)^{1 / 2}} \leqq C_{1}
$$

and

$$
C_{0}^{\prime} \leqq \lim _{\left|t-t^{\prime}\right|=h \rightarrow 0 ; 0 \leqq t . t^{\prime} \leqq 1} \frac{\left|X(t)-X\left(t^{\prime}\right)\right|}{\left(2 \sigma^{2}(h) \log 1 / h\right)^{1 / 2}} \leqq C_{1}
$$

where $f(h)$ is some function dependent upon $\sigma^{2}(h)$ and $C_{0}, C_{0}^{\prime}, C_{1}, C_{1}^{\prime}$ are constants greater than zero. For some processes $C_{0}, C_{0}^{\prime}$ and $C_{1}, C_{1}^{\prime}$ can be taken to be equal to 1 .

We begin with the Hölder conditions at a point. The results on the lower bound restrict us to those processes for which $\sigma^{2}(h)$ is concave for $h \in[0, \delta]$ for some $\delta>0$. Referring to Theorem 1 , the bound $f_{2}(h)$ will be of most interest to us, whereas $f_{2}(h)$ of Theorem 3 will be used, in most cases, to obtain the upper bound (we shall denote this as $\bar{f}_{2}(h)$ to avoid confusion). Therefore, the problem is to seek those values of $\sigma^{2}(h)$ for which

$$
1 / g^{\prime}(\log 1 / h) \leqq \text { Const } \frac{1}{\sigma(u)} \int_{0}^{h} \frac{\sigma(u)}{u} d u .
$$


Recall that the conditions of Theorems 1 and 3 limit our attention to those cases where $\int_{0}^{h}(\sigma(u) / u) d u<\infty$ and where $1 / g^{\prime}(\log 1 / h)$ is at least as large as $\log _{2} 1 / h$.

The functions in (3.3) are related as follows

$$
\frac{1}{\sigma(h)} \int_{0}^{h} \frac{\sigma(u)}{u} d u=\int_{0}^{h} \frac{1}{g^{\prime}(\log 1 / u)} d \frac{\sigma(u)}{\sigma(h)}
$$

Thus $\bar{f}_{2}(h)$ is an averaging of $f_{2}(h)$. Clearly,

$$
\min _{h \leqq t} \frac{1}{\sigma(h)} \int_{0}^{h} \frac{\sigma(u)}{u} d u \geqq \inf _{h \leqq t} 1 / g^{\prime}(\log 1 / h) .
$$

Also, if $f_{2}(h)$ is bounded above by certain smooth functions, so is $\bar{f}_{2}(h)$, as the following lemma demonstrates:

LemMA 5. Suppose $1 / g^{\prime}(\log 1 / h) \leqq a(\log 1 / h)^{r}, 0<r \leqq 1$. Then

$$
\frac{1}{\sigma(h)} \int_{0}^{h} \frac{\sigma(u)}{u} d u \leqq \frac{1}{1-\left(\operatorname{ar} /(\log 1 / h)^{1-r}\right)} a(\log 1 / h)^{r}
$$

where $a<1$ if $r=1$.

Proof. The proof follows from the inequality $1 / g^{\prime}(\log 1 / u)=\sigma(u) / u \sigma^{\prime}(u)$ $\leqq a(\log 1 / u)^{r}$.

We next show that $f_{2}(h)$ and $\vec{f}_{2}(h)$ have the same average value in the following sense: Make a scale change by setting $s=\log 1 / h$ so that $f_{2}(h)=1 / g^{\prime}(s)$ and $\vec{f}_{2}(h)$ $=F(s)=e^{g(s)} \int_{s}^{\infty} e^{-g(u)} d u$. We have now Lemma 6 .

LEMMA 6. Let $s=\log 1 / u$ and $1 / g^{\prime}(s) \leqq a s^{r}, 0<r \leqq 1$ with $a<1$ if $r=1$. Then for sufficiently large $N$

$$
1 \leqq \frac{1}{N} \int^{N} g^{\prime}(s) F(s) d s \leqq 1+\frac{a}{N^{1-r}}
$$

Proof. Note that $F^{\prime}(s)=g^{\prime}(s) F(s)-1$; Lemma 5 is also used.

It is difficult to say any more on the relationship between $(1 / \sigma(h)) \int_{0}^{h}(\sigma(u) / u) d u$ and $1 / g^{\prime}(\log 1 / h)$ without restricting the behavior of $1 / g^{\prime}(\log 1 / h)$. Examples of concave $\sigma^{2}(h)$ functions can be found for which $1 / g^{\prime}(\log 1 / h)$ oscillates very strongly. Even requiring that $1 / g^{\prime}(\log 1 / h)$ is monotonically increasing does not insure that a constant $C$ can be found such that $(1 / \sigma(h)) \int_{0}^{h} \sigma(u) / u d u \leqq C 1 / g^{\prime}(\log 1 / h)$. However, we can obtain such a relationship if we require that $1 / g^{\prime}(\log 1 / h)$ is sufficiently smooth. Writing $1 / g^{\prime}(s)$ where $s=\log 1 / h$, our criterion of smoothness is that $1 / g^{\prime}(s)$ is a regularly varying function.

A function $f(x)$ is said to be regularly varying [9], [10] if it can be put in the form $x^{p} L(x)$ where $(L(x)>0)$ is slowly varying, i.e., $\lim _{x \rightarrow \infty} L(t x) / L(x)=1$ for any $t>0$. Requiring that $1 / g^{\prime}(s)$ is regularly varying means that it has the form $s^{r} L(s)$, $0 \leqq r \leqq 1$. As in Lemma 5 , care must be taken when $r=1$. In this case lim $\sup _{s \rightarrow \infty} L(s)$ $<1$. 
LEMMA 7. Let $1 / g^{\prime}(s)$ be a regularly varying function with exponent $0 \leqq r \leqq 1$.

Case 1. If $r<1, g^{\prime}(s) F(s) \sim 1$ (i.e., $\lim _{s \rightarrow \infty} g^{\prime}(s) F(s)=1$ ).

Case 2. Define $\chi_{s}(a)=\exp \{-(g(a s)-g(s))\}, a>1$. When $r<1, \lim _{s \rightarrow \infty} \chi_{s}(a)=0$. If $\lim _{s \rightarrow \infty} \chi_{s}(a)=\chi(a)$ then

$$
g^{\prime}(s) F(s) \sim \lim _{a \rightarrow 1} \frac{1-\chi(a)}{1-a \chi(a)}
$$

Case 3. If $1 / g^{\prime}(s)<\beta s, \beta<1$, then for any $\varepsilon>0$, $a>1$

$$
(1-\varepsilon) \leqq g^{\prime}(s) F(s) \leqq a(1+\varepsilon) /\left(1-a^{1-1 / \beta}\right)
$$

for s sufficiently large.

Proof. If $1 / g^{\prime}(s)=s^{r} L(s)$, then $g(s)=s^{1-r} \tilde{L}(s)$, where $\tilde{L}$ is some other slowly varying function. Thus, for $a>1$

$$
g(a s)-g(s)=s^{1-r} \tilde{L}(a s)\left[a^{1-r}-\tilde{L}(s) / \tilde{L}(a s)\right] .
$$

For any $a>1$ and $s$ sufficiently large, the term in the brackets is positive. Thus, as long as $r<1, \chi_{s}(a) \rightarrow 0$. When $r=1, g(s)$ itself is slowly varying and $\chi_{s}(a)$ need not have a limit. However, in many cases it does, e.g., if $g(s)=\alpha \log s$. Note that when $\chi_{s}(a)$ does not have a limit we can still make use of the bound on $1 / g^{\prime}(s)$ in Case 3 to bound $\chi_{s}(a)$ above by $a^{-1 / \beta}$.

We write

$$
\begin{aligned}
g^{\prime}(s) F(s) & =g^{\prime}(s) e^{g(s)} \int_{s}^{\infty} e^{-g(u)} d u \\
& =-e^{g(s)} \int_{s}^{a s} \frac{g^{\prime}(s)}{g^{\prime}(u)} d e^{-g(u)}+a g^{\prime}(s) e^{g(s)} \int_{s}^{\infty} \chi_{u}(a) e^{-g(u)} d u .
\end{aligned}
$$

Consider $g^{\prime}(s) / g^{\prime}(u)$ for $s \leqq u \leqq a s$. This is equal to $(u / s)^{r} L(u) / L(s) \leqq a^{r}(1+\varepsilon)$ since if we write $u=b s, L(b s) / L(s) \rightarrow 1$ uniformly. (This is a fact about slowly varying functions.) When $\chi_{s}(a) \rightarrow \chi(a)$

$$
g^{\prime}(s) F(s) \sim a^{r}(1-\chi(a))+a \chi(a) g^{\prime}(s) F(s) .
$$

This gives Case 2, and Case 1 when $r<1$ since $\chi(a)=0$. Case 3 is easily obtained by substituting the upper bound for $\chi_{s}(a)$. For a given value of $\beta,(3.5 a)$ can be minimized with respect to $a$.

The conditions imposed in Lemma 6 can be used to describe the function $g(s)$. If $1 / g^{\prime}(s)=s^{r} L(s)$, then $g^{\prime}(s)=\tilde{L}(s) / s^{r}(\tilde{L}(s)=1 / L(s)$ is a slowly varying function). Therefore, $g(s)$ can be written in the following form [10, p. 274],

$$
g(s)=\text { Const } s^{1-r} \exp \left\{\int^{s} \frac{\varepsilon(t)}{t} d t\right\}, \quad \varepsilon(t) \rightarrow 0 .
$$

Furthermore, the fact that $\sigma(h)=\exp \{-g(\log 1 / h)\}$ is concave for $h$ sufficiently small necessitates that $\varepsilon^{\prime}(t)<1-r$ in those cases when $r<1$. When $r=1$ other conditions are added; they can be easily obtained from (3.7). In Case 2 the 
requirement that $\lim _{s \rightarrow \infty} \chi_{s}(a)=\chi(a)>0$ implies that $e^{-g(s)}=\left(1 / s^{\alpha}\right) L(s)$ and the requirement that $1 / g^{\prime}(s)<s$ implies that $\alpha>1$. Note that this requirement is consistent with Corollary 2.

The following lemma displays another class of processes for which a statement like (3.1) can be obtained.

LemMA 8. Suppose $1 / g^{\prime}(\log 1 / h) \leqq a \log 1 / h(a<1)$ and that $1 / g^{\prime}(s)$ is concave in $s(s=\log 1 / h)$, for $s$ sufficiently large, then

$$
\frac{1}{\sigma(h)} \int_{0}^{h} \frac{\sigma(u)}{u} d u \leqq \frac{1}{1-a} \frac{1}{g^{\prime}(\log 1 / h)} .
$$

Proof. Since $1 / g^{\prime}(s) \leqq a s$ for $a<1$ and $1 / g^{\prime}(s)$ is concave in $s$, then for sufficiently large $s d / d s\left(1 / g^{\prime}(s)\right) \leqq a$. Thus

$$
\begin{aligned}
\frac{1}{\sigma(h)} \int_{0}^{h} \frac{\sigma(u)}{u} d u= & \frac{1}{\sigma(h)} \int_{\log 1 / h}^{\infty} e^{-g(v)} d v=\frac{1}{g^{\prime}(\log 1 / h)} \\
& +\frac{1}{\sigma(h)} \int_{\log 1 / h}^{\infty} e^{-g(s)} d / d s\left(\frac{1}{g^{\prime}(s)}\right) d s
\end{aligned}
$$

from which the proof of the lemma follows.

The following theorem summarizes the above results.

THEOREM 5. Let $X(t)$ be a separable, real-valued Gaussian process with stationary increments for which $E\left\{(X(t+h)-X(t))^{2}\right\}=\sigma^{2}(h)$, where $\sigma^{2}(h)$ is concave for $h \in[0, \delta]$ for some $\delta>0$. Define $g(\log 1 / h)=-\log \sigma(h)$ and assume that $1 / g^{\prime}(s)$ is a regularly varying function of $s$ such that $\log s \leqq 1 / g^{\prime}(s) \leqq \beta s, \beta<1$. Then with probability 1

$$
C_{0} \leqq \limsup _{h \rightarrow 0} \frac{|X(t+h)-X(t)|}{\left(2 \sigma^{2}(h) f(h)\right)^{1 / 2}} \leqq C_{1}
$$

$C_{0}>0, C_{1}<\infty$, but not necessarily the same for each process. The function $f(h)$ can be either $1 / g^{\prime}(\log 1 / h)$ or $(1 / \sigma(h)) \int_{0}^{h}(\sigma(u) / u) d u$.

If $1 / g^{\prime}(s)$ is also monotone as $s \rightarrow \infty$, then it need only be bounded above, i.e., $1 / g^{\prime}(s) \leqq \beta s, \beta<1$. Equation (3.8) holds with $f(h)$ replaced by

$$
f^{*}(h)=\max \left(\log _{2} 1 / h, f(h)\right) .
$$

Proof. The proof is obtained from Lemma 7 and the preceding remarks, except for the last part which follows from Case 3, in Theorem 1.

Finally, all the results above required that $\int_{0}^{h}(\sigma(u) / u) d u<\infty$. However, as we have stated, there are processes with continuous paths for which this integral diverges. The following lemma applies to some of these processes.

LEMmA 9. Suppose $1 / g^{\prime}(\log 1 / h)<\beta \log 1 / h, \beta<2$. Then

$$
\left[\frac{1}{\sigma(h)} \int_{(2 \log 1 / h)^{1 / 2}}^{\infty} \sigma\left(e^{-x^{2}}\right) d x\right]^{2}<\frac{\text { Const } \log 1 / h}{(1-\beta / 2)} .
$$


Proof. The proof is similar to that in Lemma 5.

Theorem 5 and Lemma 9 give rise to the following examples:

$$
\begin{aligned}
& \sigma(h)=\exp \left(-a \log _{2} 1 / h\right)=1 /(\log 1 / h)^{a} \quad\left(a>\frac{1}{2}\right), \\
& \sigma(h)=\exp \left(-a(\log 1 / h)^{\gamma_{1}}\left(\log _{2} 1 / h\right)^{\gamma_{2}} \ldots\left(\log _{n} 1 / h\right)^{\gamma_{n}}\right) .
\end{aligned}
$$

$\left(0<\gamma_{1}<1\right), \gamma_{2}, \gamma_{3}, \ldots, \gamma_{n}$ are arbitrary real numbers. The corresponding $1 / g^{\prime}(\log 1 / h)$ functions are,

$$
1 / g^{\prime}(\log 1 / h)=(\log 1 / h) / a
$$

and

$$
\begin{aligned}
\frac{1}{g^{\prime}(\log 1 / h)}= & \frac{(\log 1 / h)^{1-\gamma_{1}}}{a \gamma_{1}\left(\log _{2} 1 / h\right)^{\gamma_{2}}\left(\log _{3} 1 / h\right)^{\gamma_{3}} \cdots\left(\log _{n} 1 / h\right)^{\gamma_{n}}} \\
& + \text { terms asymptotically small compared to the first term. }
\end{aligned}
$$

Additional examples can be obtained from using the remarks following Lemma 7 and from Theorem 6.

We have not been overly concerned with the values of the constants $C_{0}$ and $C_{1}$ in (3.1) because, although they can be sharpened in many cases, it seemed unimportant to do so unless they could be made to be equal. There are instances however, when this is possible. If $1 / g^{\prime}(\log 1 / h)=o\left(\log _{2} 1 / h\right)$, it follows that $(1 / \sigma(t)) \int_{0}^{t} \sigma(u) / u d u$ does also. This implies that the function $f_{2}(h)$ that appears in Theorem 3 is $\log _{2} 1 / h$. Tracing through the steps of the proof of Theorem 3 we can improve upon the constant to obtain

$$
P\left\{\limsup _{h \rightarrow 0} \frac{|X(t+h)-X(t)|}{\left(2 \sigma^{2}(h) \log _{2} 1 / h\right)^{1 / 2}} \leqq 1\right\}=1 .
$$

For the lower bound, Case 1 of Theorem 1 applies

$$
P\left\{\frac{\left|X\left(t+h_{k}\right)-X\left(t+h_{k+1}\right)\right|}{\left(2 \sigma^{2}\left(h_{k}\right) \log _{2} 1 / h_{k}\right)^{1 / 2}} \geqq 1-\sqrt{ } \theta \text { i.o. }\right\}=1 .
$$

Simply by applying the triangle inequality in (3.12), we can obtain $C_{0}=1 / 2$ in (3.1). However, in order to obtain $C_{0}=1$ we must add the restriction that $\sigma^{2}(h) \leqq h^{2 \alpha}$ for some $\alpha>0$. (By "must" we do not mean mathematical necessity. It is simply that the author needs this restriction in order to complete the proof. It seems that one ought to be able to argue probabilistically that (3.12) implies the same result with $h_{k+1}$ replaced by 0 , at least for an infinite number of $k$ 's.)

If we choose $\sigma^{2}\left(h_{k}\right)=\theta^{k}$ we see that

$$
\begin{aligned}
P\left\{\frac{\left|X\left(t+h_{k}\right)-X\left(t+h_{k+1}\right)\right|}{\left(2 \sigma^{2}\left(h_{k}\right) \log _{2} 1 / h_{k}\right)^{1 / 2}} \geqq 1-\sqrt{ } \theta\right\} \\
\geqq \frac{\text { Const }}{\left(\log _{2} 1 / h_{k}\right)^{1 / 2}} \exp \left\{-\frac{(1-\sqrt{ } \theta)^{2}}{1-\theta} \log _{2} 1 / h_{k}\right\}
\end{aligned}
$$


because the concavity of $\sigma^{2}$ implies that $\sigma^{2}\left(h_{k}-h_{k+1}\right) \geqq \sigma^{2}\left(h_{k}\right)-\sigma^{2}\left(h_{k+1}\right)$. Since $\sigma^{2}(h) \leqq h^{2 \alpha}$, then for some $\alpha>0, h_{k} \geqq \theta^{k / 2 \alpha}$. Thus, (3.13) is a term of a divergent series. By the Chung-Erdös lemma and the triangle inequality, we have for each path $X$ (with the possible exception of a set of paths of measure zero) the following inequality for an infinite number of $h_{k}$ 's:

$$
\left|X\left(t+h_{k}\right)-X(t)\right| \geqq(1-\sqrt{ } \theta)\left(2 \sigma^{2}\left(h_{k}\right) \log 1 / h_{k}\right)^{1 / 2}-\left|X\left(t+h_{k+1}\right)-X(t)\right| .
$$

Also, again excepting a set of paths of measure zero, for each path $X$ above and for any $\varepsilon>0$ we can find a sufficiently small $k^{\prime}$ such that the following holds by (3.11) for each of the above $h_{k}$ 's, as long as $k \geqq k^{\prime}$ :

$$
\left|X\left(t+h_{k}\right)-X(t)\right| \geqq(1-\sqrt{ } \theta)\left(2 \sigma^{2}\left(h_{k}\right) \log _{2} 1 / h_{k}\right)^{1 / 2}-(1+\varepsilon)\left(2 \sigma^{2}\left(h_{k+1}\right) \log _{2} 1 / h_{k+1}\right)^{1 / 2} .
$$

Note that $h_{k} \geqq \theta^{k / 2 \alpha}$ and also since $\sigma^{2}(h)$ is the incremental covariance of a stationary Gaussian process $h_{k}^{3} \leqq \sigma^{2}\left(h_{k}\right)=\theta^{k}$. These observations show that $\log _{2} 1 / h_{k+1}$ $\leqq$ Const $\log _{2} 1 / h_{k}$. Thus, since $\sigma^{2}\left(h_{k}\right)=\theta^{k}$ we can find a $\theta_{0}$, sufficiently small, so that for all $0<\theta \leqq \theta_{0}$,

$$
\left|X\left(t+h_{k}\right)-X(t)\right| \geqq(1-2 \sqrt{ } \theta)\left(2 \sigma^{2}\left(h_{k}\right) \log _{2} 1 / h_{k}\right)^{1 / 2} .
$$

We have proved the following theorem:

THEOREM 6. Let $X(t)$ be a separable, real-valued Gaussian process, with stationary increments for which $E\left\{(X(t+h)-X(t))^{2}\right\}=\sigma^{2}(h)$, where $\sigma^{2}(h)$ is concave for $h \in[0, \delta]$ for some $\delta>0$. Let $1 / g^{\prime}(\log 1 / h)=o\left(\log _{2} 1 / h\right)$ and $\sigma^{2}(h) \leqq h^{2 \alpha}$ for some $\alpha>0$. Then

$$
P\left\{\limsup _{h \rightarrow 0} \frac{|X(t+h)-X(t)|}{\left(2 \sigma^{2}(h) \log _{2} 1 / h\right)^{1 / 2}}=1\right\}=1 .
$$

We shall now consider uniform Hölder conditions. From Theorems 2 and 4, we have the following:

THEOREM 7. Let $X(t)$ be a separable, real-valued Gaussian process with stationary increments. Let $E\left\{(X(t+h)-X(t))^{2}\right\}=\sigma^{2}(h)$, where $\sigma^{2}(h)$ is concave for $h \in[0, \delta]$ for some $\delta>0$. Assume also that $1 / g^{\prime}(\log 1 / h)=o(\log 1 / h)$. Then

$$
P\left\{\limsup _{\left|t-t^{\prime}\right|=h \rightarrow 0 ; 0 \leqq t, t^{\prime} \leqq 1} \frac{\left|X\left(t^{\prime}\right)-X(t)\right|}{\left(2 \sigma^{2}(h) \log 1 / h\right)^{1 / 2}}=1\right\}=1 .
$$

for any $\varepsilon>0$.

If we compare Theorem 7 to Theorem 5, taking account of (3.9) and (3.10), we see that the uniform Hölder condition of the processes that we are considering has the same form as Levy's uniform Hölder condition for Brownian motion. However, in our results on the local Hölder condition, other functions besides the iterated logarithm are introduced.

We now extend (3.2) to those processes for which $C_{0}=C_{1}=1$ are replaced by constants dependent on $\sigma^{2}(h)$. By Theorem 2, 1 is always a lower bound. Examining 
the proof of Theorem 4 and using Lemma 9, we can obtain the following corollary to Theorem 7:

Corollary 3. Let $X(t)$ be a separable, stationary, Gaussian process for which $\sigma^{2}(h)$ is concave in $[0, \delta]$ for some $\delta>0$. Assume also that $1 / g^{\prime}(\log 1 / h)<a \log 1 / h$ $(a<2)$; then the following event has probability 1 .

$$
1 \leqq \lim _{\left|t-t^{\prime}\right|=h \rightarrow 0 ; 0 \leqq t, t^{\prime} \leqq 1} \frac{\left|X\left(t^{\prime}\right)-X(t)\right|}{\left(2 \sigma^{2}(h) \log 1 / h\right)^{1 / 2}} \leqq \text { Const }\left(\frac{1}{2-a}\right) .
$$

In some cases the constant on the left can also be increased. In proving this we use the more general form of the Chung-Erdös lemma (i.e., $\sum_{j \neq k} P\left(B_{j}, B_{k}\right) \leqq$ $\left.\sum_{j \neq k} P\left(B_{j}\right) P\left(B_{k}\right) j, k=1, \ldots, n\right)$ instead of $P\left(B_{j}, B_{k}\right) \leqq P\left(B_{j}\right) P\left(B_{k}\right)$ and take the increments $X\left(t+t_{k}\right)-X\left(t+t_{k+1}\right)$ so that they overlap in time (i.e., $t_{k}>t_{k+2}$ $\left.>t_{k+1}>t_{k+3}\right)$. This remains to be studied further.

The bound $1 / g^{\prime}(\log 1 / h)<a \log 1 / h(a<2)$ was used to bound the integrals in Fernique's lemma. However, an essential part of the proof of Theorem 7 and Corollary 3 is that $\sigma^{2}(h) \log 1 / h$ is monotonically increasing in $h$. This condition is equivalent to $1 / g^{\prime}(\log 1 / h) \leqq 2 \log 1 / h$. Thus, even if Fernique's lemma could be sharpened, or if a better bound for the integrals could be found, it could not be incorporated into our proof so as to extend Corollary 3 (except perhaps to replace $a<2$ by $a \leqq 2)$.

If $\sigma^{2}(h)$ is concave, then $1 / g^{\prime}(\log 1 / h) \geqq 2 \log 1 / h$ is a sufficient condition that the corresponding Gaussian process has discontinuous sample paths (see Corollary 2). Thus, for continuous processes, if $1 / g^{\prime}(\log 1 / h)$ exceeds $2 \log 1 / h$ it must be less than this value also. As we remarked above, $1 / g^{\prime}(\log 1 / h)$ can oscillate very strongly. In fact, we can find a concave $\sigma^{2}(h)$ corresponding to a continuous Gaussian process, for which $1 / g^{\prime}\left(\log 1 / h_{k}\right)$ has discontinuities at a sequence $h_{k} \rightarrow 0$ for which

$$
1 / g^{\prime}\left(\log 1 / h_{k}\right)=1 / 3 \sqrt{ } h_{k}
$$

and $1 / g^{\prime}\left(\log 1 / h_{k}-\right)=4 / 3$. In this case $\sigma^{2}(h) \log 1 / h$ is probably not the proper denominator for $|X(t+h)-X(t)|$ if we seek a result like (3.14).

Therefore, although we have described the local behavior of a wide class of Gaussian processes, we have had to restrict ourselves to those processes for which $E\left\{(X(t+h)-X(t))^{2}\right\}$ satisfies smoothness conditions even stronger than concavity in $[0, \delta]$ for some $\delta>0$. It is not clear precisely what the denominator of $|X(t+h)-X(t)|$ should be for results of the form of (3.1) and (3.2), but with the restrictions on $1 / g^{\prime}(\log 1 / h)$ removed. It might be that in these cases it is no longer helpful to try to consider the denominator as having the form $\sigma^{2}(h) f(h)$.

\section{REFERENCES}

1. K. L. Chung and P. Erdös, On the application of the Borel-Cantelli lemma, Trans. Amer. Math. Soc. 72 (1952), 179-189.

2. K. L. Chung, P. Erdös and T. Sirao, On the Lipschitz's condition for Brownian motion, J. Math. Soc. Japan 11 (1959), 263-274. 
3. X. Fernique, Continuité des processus Gaussiens, C. R. Acad. Sci. Paris 258 (1964), 6058-6060.

4. M. Loève, Probability theory, 3rd ed., Van Nostrand, Princeton, N. J., 1963.

5. T. Sirao, On the uniform continuity of Wiener process, J. Math. Soc. Japan 6 (1954), 332-335.

6. Yu K. Belyaev, Continuity and Hölder's conditions for sample functions of stationary Gaussian processes, Fourth Berkeley Sympos. Math Statist. and Prob., Vol. 2, Univ. of California Press, Berkeley, Calif., 1961, pp. 23-33.

7. M. B. Marcus, Gaussian process with stationary increments possessing discontinuous sample paths, The RAND Corporation, RM-5226-PR, December, 1966; Pacific J. Math (to appear).

8. P. Levy, Théorie de l'addition des variables aléatoires, Gauthier-Villars, Paris, 1954.

9. J. Karamata, Sur un mode de croissance régulière, Mathematica (Cluj) 4 (1930), 38-53.

10. W. Feller, An introduction to probability theory and its applications, Vol. 2, Wiley, New York, 1966.

11. G. A. Hunt, Random Fourier transforms, Trans. Amer. Math. Soc. 71 (1951), 38-69.

12. H. Cramer and M. R. Leadbetter, Stationary and related stochastic processes, Wiley, New York, 1967, 187-189.

THE RAND CORPORATION,

Santa Monica, California 